\title{
Upper Limits on the X-ray Emission of "Uranium" Stars
}

\author{
Eric M. Schlegel \\ Harvard-Smithsonian Center for Astrophysics
}

\begin{abstract}
A paper by Qian \& Wasserburg suggests the optical absorption lines of uranium observed in the spectra of ultra-metal-poor stars (defined as $[\mathrm{Fe} / \mathrm{H}]<-3$ ) arise from contamination from a supernova in a binary star system. Assuming the binary survived the explosion, a collapsed compact object may be present and implying potential accretion processes with accompanying $\mathrm{X}$-ray emission. Upper limits on X-ray emission from an accreting compact object are described here.

Subject headings:
\end{abstract}

\section{Introduction}

Datings of radioactive materials provide the firmest ages known. In the case of long-lived heavy elements such as thorium and uranium, derived age values encompass the estimated age of the Galaxy.

Ultra-metal poor stars, defined as stars with very low abundances of heavy elements $([\mathrm{Fe} / \mathrm{H}] \sim$ 3) and largely confined to the Galactic halo, evidently represent the earliest generation of stars formed as the Galactic disk was still collapsing (e.g., Qian \& Wasserburg 2001a; Cayrel et al. 2001). As such, they are largely devoid of contamination by subsequent generations of supernovae and star formation that occurred within the disk.

Recently, observations of three stars, uncovered from optical surveys of ultra-metal-poor stars (Ryan, Norris, \& Beers 1996 and references therein), revealed absorption lines of r-process elements. BPS CS31082-001, hereafter CS31082, exhibits an optical absorption line from uranium at $3889.57 \AA$ which yields an abundance of $\log (\mathrm{U} / \mathrm{H})$ $=-13.7 \pm 0.14 \pm 0.12($ random + systematic $)$ and an estimated age for the star of $12.5 \pm 3$ Gyr (Cayrel et al. 2001). BPS CS22892-052, hereafter CS22892, also exhibits optical absorption lines from heavy elements, particularly thorium (absorption line at $4019 \AA$ A , Sneden et al. 2000). Both stars show absorption lines from r-process elements enhanced relative to Fe. Cowan et al. (2002) report the detection of a weak optical line in $\mathrm{BD}+17^{\circ} 3248$, hereafter BD17, at $3589.6 \AA$ from U II. Other stars undoubtedly show similar line features of r-process elements.

Uranium and other neutron-rich nuclei have long been believed to be produced in the explosive nucleosynthesis accompanying a supernova (e.g., Hillebrandt, Nomoto, \& Wolff 1984). Briefly, the r-process is a chain of neutron captures required to explain neutron-rich nuclides for species above atomic mass 70 . Successful production requires very high neutron densities $\left(\mathrm{N}_{\mathrm{n}}>10^{19} \mathrm{~cm}^{-3}\right)$ and high temperature $\left(\mathrm{T} \sim 10^{9} \mathrm{~K}\right)$ for the capture process to proceed more rapidly than $\beta$ decay. The site of the r-process is not well-defined, but the required conditions essentially demand an association with a supernova.

Subsequent research raised significant questions about the prompt mechanism and spurred searches for other sites. One mechanism currently enjoying wide support sites the r-process in the $\nu$-heated ejecta of the newborn neutron star (Woosley \& Hoffman 1992; Meyer et al. 1992; Woosley et al. 1994; Otsuki et al. 2000; Terasawa et al. 2001). Other sites include merging neutron stars (Freiburghaus, Rosswog, \& Thielemann 1999) and collapsing ONeMg cores (Wheeler, Cowan, Hillebrandt 1998). Sumiyoshi et al. (2001) resurrected the prompt mechanism using new, slower $\mathrm{e}^{-}$-capture rates. A recent paper by Meyer 
(2002) shows that an excess of neutrons may not be necessary for the $\mathrm{r}$ process to proceed.

Qian \& Wasserburg (2001b) argued that the presence of r-processed material in ultra-metalpoor stars suggested the possible presence of a compact binary companion that formed after the detonation of the supernova. Their argument is based upon a theory for element production developed during the past few years (summarized in Qian \& Wasserburg 2000). Based upon their study of the cosmic abundances, they postulate the existence of high- and low-frequency Type II (core collapse) supernovae. The class of progenitors of the high-frequency supernovae detonate quickly, on the time scale of stellar evolution, or with a rate of approximately $10^{-7} \mathrm{yr}^{-1}$. The lowfrequency supernovae detonate less often, at a rate of approximately $10^{-8} \mathrm{yr}^{-1}$.

The nucleosynthetic products generated by the two types of supernovae differ, with the frequent supernovae producing the majority of the heavy r-process nuclei (with mass number $>135$ ) while the less-frequent supernovae account for the majority of the lighter r-process nuclei (mass number $<135)$. Evidence in support for the bifurcation in r-process production was presented by Sneden et al. (2000) who showed that the abundances of CS22892-052 followed the solar r-pattern for elements heavier than barium, but were difficient for elements near $\mathrm{Ag}(\mathrm{A}=107)$.

Wasserburg \& Qian (2000) argue that the presence of heavy r-process elements in ultra-metalpoor stars with low Fe abundances implies that the high-frequency supernovae can produce r-process material without the existence of a large abundance of iron. The observations of uranium in CS31082-001 provide evidence in support for their argument (Qian \& Wasserburg 2001b).

If a compact companion did form and if the binary survived the explosion, the companion's presence should be detected from the production of $\mathrm{X}$-rays from accretion processes. Sensitive X-ray observations may test for this situation.

\section{Observations}

We surveyed the entire data base of satellite X-ray data available through the HEASARC collection. No pointed observations from Einstein, ROSAT, ASCA, Chandra, or XMM-Newton exist within $60^{\prime}$ of the targets. The ROSAT All-Sky Survey $^{1}$ (RASS) provides the only measurements of the X-ray flux at their locations with exposures of at most a few hundred seconds. Table 1 lists the coordinates of the three fields and the RASS field numbers obtained from the archive.

Figures 1, 2, and 3 show the RASS fields containing each target. In each field, circles surround the objects for which X-ray counts were extracted. The labelled circles correspond to the sources listed in Table 3. In Figure 2, two of the unlabelled, unextracted sources are identified as Seyfert 1 galaxies (1RXS J221632.7-160533 northernmost; 1RXS J221300.1-171019, westernmost source).

We extracted the counts at the positions of the target stars from the data using apertures $5^{\prime}$ in radius. The background was obtained from an annulus of outer diameter $20^{\prime}$. We also extracted the mean exposure time from the corresponding location in the exposure map. Table 2 lists the extracted counts. Bayesian upper limits were determined using the prescription in Loredo (1992).

The estimated distances to two target stars fall in the range of 4-6 kpc (Beers et al. 2000). For simplicity, we adopt $5 \mathrm{kpc}$. For $\mathrm{BD}+17$, the distance is listed as $0.7 \mathrm{kpc}$ (Beers et al. 2000). The column density in the direction of the targets is low, a few $\times 10^{20} \mathrm{~cm}^{-2}$ (Table 1). Fluxes were computed from the $99 \%$ upper limits assuming a thermal spectrum of temperature $1 \mathrm{keV}$ absorbed by the Galactic columns in the direction of each source. The fluxes approximately double if the adopted temperature is $5 \mathrm{keV}$.

To provide additional confidence in our upper limits, the counts of several faint point sources positionally near the uranium stars were also extracted. The positions and counts are listed in Table 3. None of the sources is an identified X-ray object based upon a search of SIMBAD ${ }^{2}$ or $\mathrm{NED}^{3}$.

\footnotetext{
${ }^{1}$ We acknowledge with thanks the support for the RASS provided by Max-Planck Institut für Extraterrestriche Astrophysik. The research discussed here would have been impossible without that support.

${ }^{2}$ This research has made use of the SIMBAD database, operated by CDS, Strasbourg, France. SIMBAD access in the USA is provided by funding from National Aeronautics and Space Administration.

${ }^{3}$ This research has made use of the NASA/IPAC Extragalactic Database (NED) which is operated by the Jet Propul-
} 


\section{Discussion}

The ROSAT observations establish an upper limit at $\sim 1-1.5 \mathrm{keV}$ of $\sim 10^{32-33} \mathrm{ergs} \mathrm{s}^{-1}$ for an assumed distance of $5 \mathrm{kpc}$. This luminosity eliminates the presence of a black hole accreting at the Eddington rate, assuming no very high local absorption, since the expected flux from a $1 \mathrm{M}_{\odot}$ black hole would be easily detected at $5 \mathrm{kpc}$, with an estimated flux rivaling that of the Crab.

If the binary did survive the explosion and if the compact object does emit at typical Eddington values, a local high column provides an exit from the restrictive upper limit. Substantial local absorption, with column densities $>10^{22} \mathrm{~cm}^{-2}$, significantly remove $\mathrm{X}$-rays with energies below $\sim 2-2.5 \mathrm{keV}$. As the ROSAT PSPC had decreasing effective area above $\sim 2 \mathrm{keV}$, the sensitivity to hard X-rays of the survey observations is considerably poorer than the flux limit mentioned above. Figure 4 illustrates the column density-luminosity relation that yields no detection in the ROSAT bandpass.

Other compact objects may exist in the assumed binaries, regardless of whether we understand the overall evolutionary scenario. In addition, quiescent levels of some $\mathrm{X}$-ray binaries in elliptical orbits (e.g., 4U0115+63) are low with a typical $\mathrm{L}_{\mathrm{X}}$ of $\lesssim 2 \times 10^{32} \mathrm{erg} \mathrm{s}^{-1}$ (Tamura et al. 1992). Persistent X-ray binaries often have low $\mathrm{L}_{\mathrm{X}}$, too. For example, $\mathrm{X}$ Per contains a neutron star, but has a total X-ray luminosity in the 2$10 \mathrm{keV}$ band of $9_{-5}^{+8} \times 10^{34} \mathrm{erg} \mathrm{s}^{-1}$ (Schlegel et al. 1993).

With relatively high upper limits, explanations for a non-detection abound. These include the failure of the assumption that the binary survived the explosion, "off" states in wind- or Roche lobeaccreting systems, or alternative explanations to generate the r-process contaminants. A larger discussion of these issues will be presented with the expected improvement in the upper limits (see following discussion). We did search proper motion catalogs for each of the targets. Only BD +17 has a measured and published proper motion of magnitude $\sim 52$ milliarcsec $\mathrm{yr}^{-1}$; the component motion in right ascension differs between Roeser \& Bas-

sion Laboratory, California Institute of Technology, under contract with the National Aeronautics and Space Administration. tian (1988) (-34 milliarcsec $\left.\mathrm{yr}^{-1}\right)$ and Beers et al. (2000) (-46 milliarcsec $\left.\mathrm{yr}^{-1}\right)$. The proper motions of the two CS stars have apparently not as yet been published.

A sensitive observation at energies above $\sim 3$ $\mathrm{keV}$ would eliminate much of all of the local column density argument. A $10 \mathrm{ksec}$ observation with the XMM-Newton EPIC detector reaches $\mathrm{L}_{\mathrm{X}}$ $\sim 2-5 \times 10^{31} \mathrm{erg} \mathrm{s}^{-1}$, constraining nearly all compact binary scenarios including cataclysmic variables (magnetic CV AM Her: $\mathrm{L}_{\mathrm{X}} \sim 2 \times 10^{33} \mathrm{erg}$ $\mathrm{s}^{-1}$; dwarf nova CV SS Cyg: $\mathrm{L}_{\mathrm{X}} \sim 1 \times 10^{32} \mathrm{erg} \mathrm{s}^{-1}$; Warner (1995)) and neutron star wind accretors (e.g., 4U1700+24, accreting from a red giant at $\dot{M} \sim 10^{-9} \mathrm{M}_{\odot} \mathrm{yr}^{-1}$, has a measured $2-10 \mathrm{keV}$ luminosity of $\sim 2 \times 10^{32} \mathrm{erg}^{-1}$, Masetti et al. 2002).

The limit reaches the upper end of the luminosity function of RS CVn stars (Shaw, Caillault, \& Schmitt 1996; Dempsey et al. 1993) but does not eliminate active binaries such as BY Dra systems (Dempsey et al. 1997) which have $\mathrm{L}_{\mathrm{X}} \sim 10^{28-30}$ $\mathrm{erg} \mathrm{s}^{-1}$. The limit also does not reach single $\mathrm{K} \mathrm{su}$ pergiants which have $\mathrm{L}_{\mathrm{X}} \sim$ few $\times 10^{29} \mathrm{erg} \mathrm{s}^{-1}$ (e.g., Hünsch et al. 1996).

Two of the targets (CS31082 and CS22892) have been approved for Cycle 2 XMM observations, so more restrictive upper limits or detections will be available shortly.

This research was supported by contract number NAS8-39073 to SAO. 


\section{REFERENCES}

Beers, T. et al. 2000, AJ, 119, 2866

Cayrel, R. et al. 2001, Nature, 409, 691

Cowan, J. J. et al. 2002, ApJ, 572, 861 (astro$\mathrm{ph} / 0202429)$

Dempsey, R. C., Linsky, J. L., Fleming, T. A., \& Schmitt, J. H. M. M. 1997, ApJ, 478, 358

Dempsey, R. C., Linsky, J. L., Fleming, T. A., \& Schmitt, J. H. M. M. 1993, ApJS, 86, 599

Freiburghaus, C., Rosswog, S., \& Thielemann, F.K. 1999, ApJ, 525, L121

Hillebrandt, W., Nomoto, K., \& Wolff, R. G. 1984, A\&A, 133, 175

Hünsch, M. et al. 1996, A\&A, 310, 801

Loredo, T. J. 1992, in Statistical Challenges in Modern Astronomy, ed. E. D. Feigelson \& G. J. Babu (New York: Springer-Verlag, Inc.), 275

Masetti, N. et al. 2001, A\&A, 382, 104 (astro$\mathrm{ph} / 0111164$ )

Meyer, B. S., 2002, PhysRevL, 89, 1101 (astro$\mathrm{ph} / 0207227)$

Meyer, B. S., Mathews, G. J., Howard, W. M., Woosley, S. E, \& Hoffman, R. D. 1992, ApJ, 399,656

Otsuki, K., Tagoshi, H., Kajino, T., \& Wanajo, S. 2000, ApJ, 533, 424

Qian, Y.-Z. \& Wasserburg, G. J. 2001a, ApJ, 552, L55

Qian, Y.-Z. \& Wasserburg, G. J. 2001b, ApJ, 559, 925

Qian, Y.-Z. \& Wasserburg, G. J. 2000, Physics Reports, 333, 77

Roeser, S. \& Bastian, U. 1988, A\&ASup, 74, 449

Ryan, S. G., Norris, J. E., \& Beers, T. C. 1996, ApJ, 471, 254

Schlegel, Eric M. et al. 1993, ApJ, 407, 744

Shaw, J. S., Caillaut, J.-P., \& Schmitt, J. H. M. M. 1996, ApJ, 461, 951
Sneden, C. et al. 2000, ApJ, 533, L139

Sumiyoshi, K., Terasawa, M., Mathews, G. J., Kajino, T., Yamada, S. \& Suzuki, H. 2001, ApJ, 562,880

Tamura, K. et al. 1992, ApJ, 389, 676

Terasawa, M., Sumiyoshi, K., Kajino, T., Mathews, G. J., \& Tanihata, I. 2001, ApJ, 562, 470

Warner, B. 1995, Cataclysmic Variables (Cambridge: Cambridge University Press)

Wasserburg, G. \& Qian, Y.-Z. 2000, ApJ, 529, L21

Wheeler, J.C., Cowan, J.J., \& Hillebrandt, W. 1998, ApJ, 493, L101

Woosley, S. E., Wilson, J. R., Mathews, G. J., Hoffman, R. D., \& Meyer, B. S. 1994, ApJ, 433, 229

Woosley, S. E. \& Hoffman, R. D. 1992, ApJ, 395, 202

This 2-column preprint was prepared with the AAS LATEX macros v5.0. 
Table 1: "Uranium" Stars and RASS Fields

\begin{tabular}{|c|c|c|c|c|c|c|c|c|c|}
\hline \multirow[b]{2}{*}{ Star } & \multicolumn{2}{|c|}{$\mathrm{J} 2000$} & \multirow{2}{*}{$\begin{array}{l}\text { ROSAT } \\
\text { Field }^{\text {a }} \\
\end{array}$} & \multirow{2}{*}{$\begin{array}{c}\mathrm{N}_{\mathrm{H}} \\
\left(\mathrm{cm}^{-2}\right) \\
\end{array}$} & \multirow[b]{2}{*}[\mathrm{Fe}/\mathrm{H}]{} & \multirow{2}{*}{$\begin{array}{r}\text { Dist } \\
(\mathrm{kpc})\end{array}$} & \multirow{2}{*}{$\begin{array}{r}\text { Rad. } \\
\text { Vel. }\end{array}$} & \multicolumn{2}{|c|}{ Proper Motion ${ }^{\mathrm{b}}$} \\
\hline & RA & Dec & & & & & & RA & Dec \\
\hline BPS CS31082-001 & $01: 29: 31.2$ & $-16: 00: 48$ & 932004 & $1.5(20)$ & -2.9 & & & & \\
\hline $\mathrm{BD}+17^{\circ} 3248$ & $17: 28: 14.5$ & $+17: 30: 36$ & 931446 & $5.9(20)$ & -2.0 & 0.703 & $-146 \pm 1$ & $-46.7 \pm 0.8$ & $-24.2 \pm 0.7$ \\
\hline BPS CS22892-052 & $22: 17: 01.5$ & $-16: 39: 26$ & 932059 & $2.6(20)$ & -3.1 & 5.7 & $10 \pm 10$ & & \\
\hline
\end{tabular}

${ }^{a}$ identification number for ROSAT All-Sky Survey field

${ }^{b}$ Values for proper motion in milliarcsec $\mathrm{yr}^{-1}$.

Table 2: Extracted X-ray Counts: "Uranium" Stars

99\% ExpTime $\quad 99 \%$

\begin{tabular}{lccrccc} 
Star & Counts \pm Err & Counts & (sec) & Rate & Flux $^{\mathrm{a}}$ & $\mathrm{Lx}^{\mathrm{b}}$ \\
\hline CS31082-001 & $2.2 \pm 2.4$ & $<6.9$ & 435 & 0.016 & $1.8(-13)$ & $5.4(32)$ \\
BD+17 3248 & $7.8 \pm 3.9$ & $<10.8$ & 350 & 0.020 & $4.9(-13)$ & $9.3(31)$ \\
CS22892-052 & $5.8 \pm 3.0$ & $<11.2$ & 277 & 0.042 & $6.0(-13)$ & $2.3(33)$ \\
\hline
\end{tabular}

${ }^{a}$ Counts converted to an unabsorbed flux assuming the Galactic column toward the sources with a 1 keV bremsstrahlung spectrum. For a temperature of $5 \mathrm{keV}$, the fluxes are all $\sim 2$ times higher.

${ }^{b} \mathrm{~L}_{\mathrm{X}}$ calculated assuming the distance in Table 1 or $5 \mathrm{kpc}$ for CS31082.

Table 3: Extracted X-ray Counts for Test Sources

\begin{tabular}{|c|c|c|c|c|c|c|}
\hline \multicolumn{3}{|c|}{ J2000 } & & \multirow{2}{*}{$\begin{array}{c}\text { ExpT } \\
(\mathrm{sec})\end{array}$} & \multirow[b]{2}{*}{ Rate } & \multirow[b]{2}{*}{ Flux } \\
\hline Field & RA & Dec & Counts \pm Err & & & \\
\hline CS31082-A & $01: 28: 59.5$ & $-15: 23: 19$ & $19.2 \pm 4.9$ & 449 & $0.043 \pm 0.011$ & $4.8 \pm 1.2(-13)$ \\
\hline CS31082-B & $01: 27: 42.9$ & $-16: 36: 30$ & $15.2 \pm 4.6$ & 394 & $0.038 \pm 0.012$ & $4.3 \pm 1.3(-13)$ \\
\hline BD+17-A & $17: 30: 34.9$ & $+17: 30: 54$ & $30.8 \pm 6.2$ & 570 & $0.054 \pm 0.011$ & $1.1 \pm 0.21(-12)$ \\
\hline CS22892-A & $22: 14: 30.2$ & $-17: 02: 03$ & $24.8 \pm 5.3$ & 308 & $0.081 \pm 0.017$ & $1.16 \pm 0.24(-12)$ \\
\hline
\end{tabular}

${ }^{a}$ Counts converted to an unabsorbed flux assuming the Galactic column toward the sources with a 1 keV bremsstrahlung spectrum. 
Fig. 1.- Field of CS31082-001. The circles are 5' in radius and surround the star's location as well as "test" sources A and B.

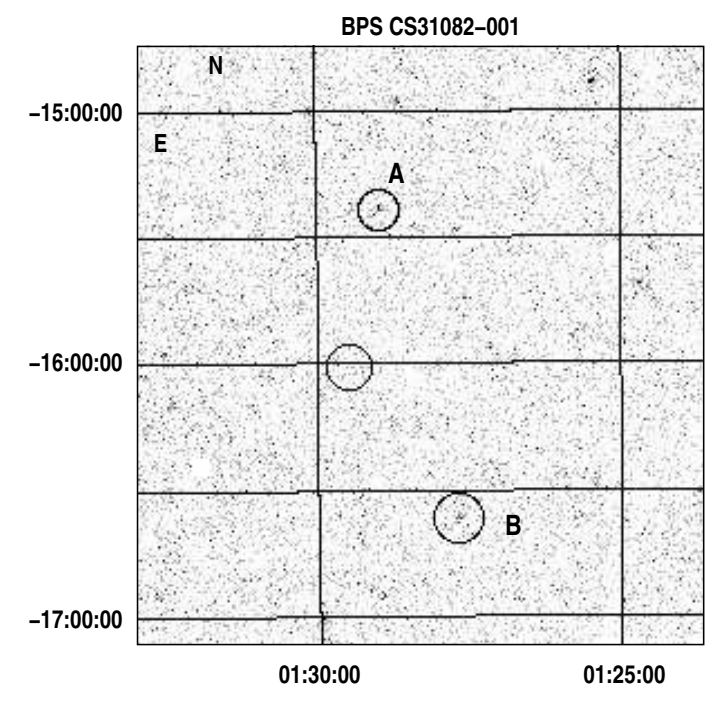

Fig. 2.- Field of CS22892-052. The circles are 5' in radius and surround the star's location as well as "test" source A. The northern- and westernmost uncircled sources are known Seyfert 1 galaxies; the southernmost is an unidentified RASS source (see text).

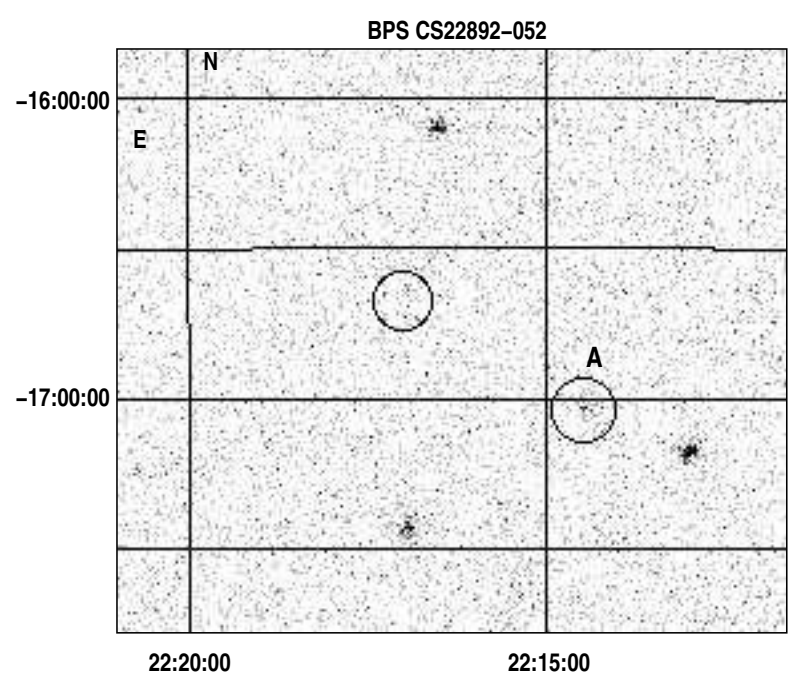


Fig. 3.- Field of $\mathrm{BD}+17^{\circ}$ 3248. The circles surround the star's location as well as test source 'A'. The source in the lower right portion of the figure is an unidentified RASS source (1RXS172534.0+160923).

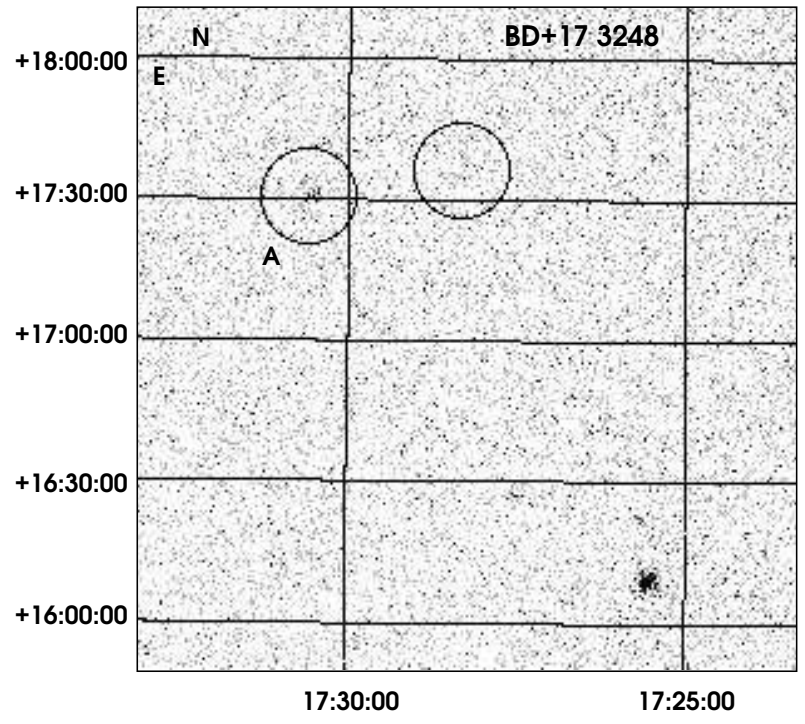


Fig. 4.- The solid and dashed curves are the locus of column densities that could absorb all the counts in the respective bands and illustrate the difficulty of obscuring hard X-ray sources from the hard band of XMM-Newton or Chandra (left vertical axis). The dashed-dotted curve shows the luminosities probed by a 25 -count detection of a $1-\mathrm{keV}$ source assumed $5 \mathrm{kpc}$ distant for exposure times on the right axis.

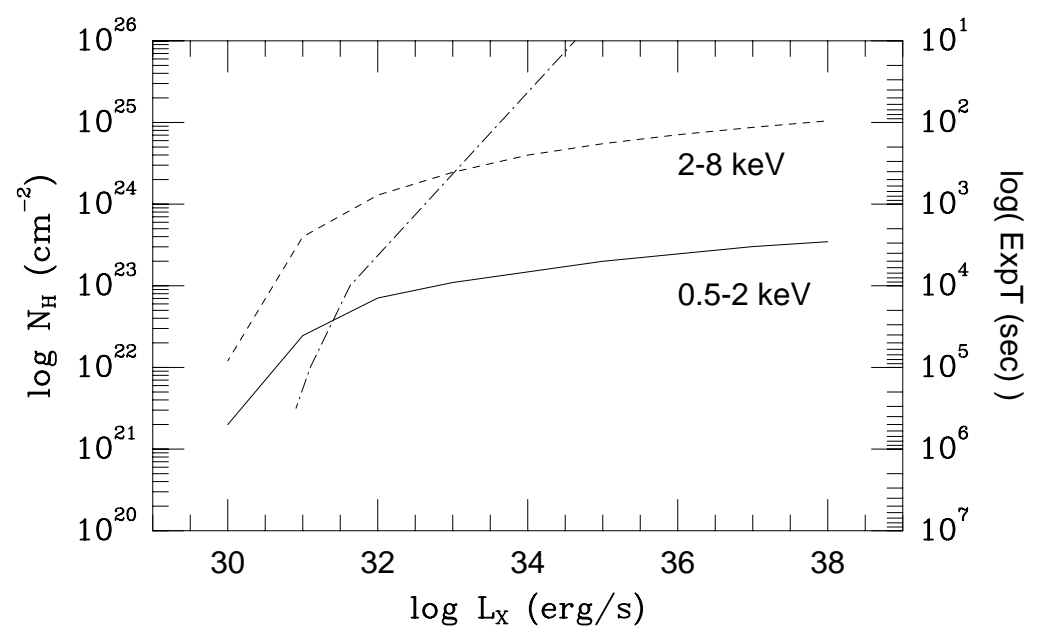

